\title{
A systematic surgical approach for the treatment of idiopathic granulomatous mastitis: a case series
}

\author{
Xiaohui Zhang ${ }^{1 \#}$, Yan Li $^{1 \#}$, Yidong Zhou ${ }^{1}$, Deshun Liu ${ }^{1}$, Linlin Chen ${ }^{2}$, Kunying Niu ${ }^{2}$, Qiang Sun ${ }^{1}$, \\ Hanyuan Huang ${ }^{1}$
}

${ }^{1}$ Department of Breast Surgery, Peking Union Medical College Hospital, Chinese Academy of Medical Science (CAMS) and Peking Union Medical College, Beijing 100730, China; ${ }^{2}$ Department of Breast Surgery, Beijing Dangdai Hospital, Beijing 100010, China

Contributions: (I) Conception and design: H Huang, X Zhang; (II) Administrative support: Q Sun, H Huang; (III) Provision of study materials or patients: X Zhang, Y Zhou, D Liu, H Huang; (IV) Collection and assembly of data: Y Li, D Liu, L Chen, K Niu; (V) Data analysis and interpretation: X Zhang, Y Li, Y Zhou; (VI) Manuscript writing: All authors; (VII) Final approval of manuscript: All authors.

\#These authors contributed equally to this work.

Correspondence to: Qiang Sun; Hanyuan Huang. Department of Breast Surgery, Peking Union Medical College Hospital, Chinese Academy of Medical Science, No. 1 Shuaifuyuan Street, Beijing 100730, China. Email: sunqpumch@163.com; huanghypumch@163.com.

\begin{abstract}
Background: Surgical resection can be performed for idiopathic granulomatous mastitis (IGM), but recurrence and tissue defects remain issues. Here we report our 6-year experience with a four-pattern surgical approach for IGM that involves the use of a random breast dermo-glandular flap (BDGF).

Methods: Sixty-eight consecutive patients with IGM were prospectively enrolled from 01/2012 and 03/2017. Based on the extent, shape, and location of the lesions, four different patterns of surgery based on BDGF were used to remove the lesion and repair the defect. Operative data (time, blood loss, and intraoperative complications), primary healing time, recurrence, and patient-reported outcomes (cosmetic outcome, and improvement in dressing change and bathing) at 2 years were evaluated.
\end{abstract}

Results: Patients' median age was 35 (range, 22-55) years. Duration of IGM was 3-22 months, with a median lesion size of 3.5 (range, 0.9-9.1) cm. The operative time was significantly longer, and blood loss was more important with the increasing lesion size (both $\mathrm{P}<0.05$ ). No significant intraoperative complications occurred. All wounds healed by primary intention. IGM relapsed in three patients (3/68, 4.4\%); they were treated successfully with a second operation. The self-evaluated cosmetic outcome was "much better" in 45 patients (66.2\%), "a little better" in 18 (26.5\%), and "same or worse" in five $(7.3 \%)$. The self-evaluated improvement in dressing change and bathing was "improved a lot" in 51 patients $(75.0 \%)$, "improved a little" in $11(16.2 \%)$, and "not improved or getting worse" in six $(8.8 \%)$.

Conclusions: The BDGF-based systematic four-pattern surgical approach is effective in the treatment of IGM. Recurrence rate is low, there are no complications, and the cosmetic results and improvement in dressing change and bathing are generally favourable.

Keywords! Granulomatous mastitis; idiopathic; recurrence; surgery; surgical flaps

Submitted Sep 09, 2019. Accepted for publication Jan 17, 2020.

doi: $10.21037 /$ gs.2020.02.06

View this article at: http://dx.doi.org/10.21037/gs.2020.02.06

\section{Introduction}

Idiopathic granulomatous mastitis (IGM) is a chronic breast inflammatory disease first reported in 1972 (1). Clinically, IGM is accompanied by breast masses, pain, and skin ulceration (2,3). It is often difficult to make a definitive diagnosis because the clinical and radiographic presentations of IGM may mimic malignancy, acute or chronic infections, and inflammatory diseases (4-9). Moreover, its etiology remains unclear and may involve several factors, including 
hormonal status, autoimmune diseases, diabetes, infections, and genetic factors (10-13).

Currently, the definitive treatment of IGM is controversial, and an optimal treatment strategy has not yet been established (14). For small lesions, conservative management such as antibiotics, corticosteroids, and methotrexate are usually adopted as the primary treatment method $(5,14-20)$. Available surgical methods can be divided into breast abscess incision and drainage, simple mass resection, extended breast mass excision, and breast segmentectomy (20). When the lesions are accompanied by large abscesses or fistula, surgical incision, and drainage, as well as fistula removal, are considered effective therapeutic methods $(5,6,21)$. Surgical excision has been suggested for severe and intractable cases $(16,18)$, but the outcomes are uncertain (17).

Extended resection is performed to remove entire lesions, and it has been widely used for IGM $(21,22)$, but the location and extent of the lesions vary from one patient to another, which represents a challenge. Many patients have to undergo multiple operations due to multiple recurrences (0-38\% of the patients) (16-18,23-26), and eventually, a certain proportion of these patients have to undergo mastectomy (18). In patients who undergo extended excision, surgery often leads to large breast tissue defects, often with healing issues $(17,18)$. Many patients may undergo repeated surgery due to the recurrence of IGM lesions, leading to unfavorable cosmetic results and psychological burden (27). In such patients, the tissue defect can be corrected using a random breast dermo-glandular flap (BDGF), which is a pedicle flap containing cutaneous, subcutaneous, and mammary gland tissues. This flap is fully nourished by arterioles in the breast region. We previously reported the favorable outcomes of BDGF in patients with recurrent and severe periductal mastitis (28).

The literature on the application of BDGF in patients with IGM is scarce, and the exact surgical strategies might vary from one patient to another. Therefore, in this study, we reported our 6-year experience with a systematic fourpattern surgical approach that involves the use of BDGF, and that covers most of the patients with IGM.

\section{Methods}

\section{Study design and patients}

This was a prospective case series of consecutive patients with IGM treated surgically between January 2012 and
March 2017 at the Breast Surgery Unit of the Peking Union Medical College Hospital (PUMCH). The study was approved by the ethics committee of the PUMCH. All patients signed an informed consent form. All procedures were in accordance with the ethical standards of the institutional and/or national research committees and with the 1964 Helsinki declaration and its later amendments or comparable ethical standards.

The inclusion criteria were: (I) $\geq 18$ years of age; (II) with clinical characteristics of IGM; (III) with presurgical pathologic diagnosis of IGM by core needle biopsy [including chronic granulomatous lobulitis with giant cell, leukocytosis, epithelioid cells, macrophages, and microabscess $(12,18)]$; and (IV) having at least one of the following conditions: (I) refractory to conservative therapy for at least 3 months; (II) recurrence after abscess incision and drainage or fistula removal; and (III) recurrence after inflamed tissue removal. The exclusion criteria were: (I) with acute infection or tuberculosis; (II) pregnant or lactating; (III) breast cancer; or (IV) refusal to participate.

\section{Patient management}

At our institution, for treatment-naive patients without abscess or ulceration, topical and oral steroid are used. If the disease is improved or cured, the patients are observed. For treatment-naive patients with abscess and ulceration, abscess incision and drainage, fistula removal, and dressing changes are performed as required. If the patients do not respond to these treatments, they are proposed surgery using the four patterns. For patients who had been operated in other hospitals but in whom the lesion could not be healed, they were treated with the four pattern surgery.

\section{Surgery}

The extent of the lesion was carefully evaluated by mammography and ultrasound, and magnetic resonance imaging (MRI) was used in patients with multiple or extensive lesions. All operations were carried out under general anesthesia, by a team composed of one professor, one fellow, and one resident. Extended excision was performed to remove the entire lesion, including the involved skin, breast abscess, fistula, and inflammatory or necrotic tissues, and with a clear margin around the breast defect, based on the histological examination. According to the location and extent of the lesion, the patients were divided into four different groups, and a specific surgical 
A

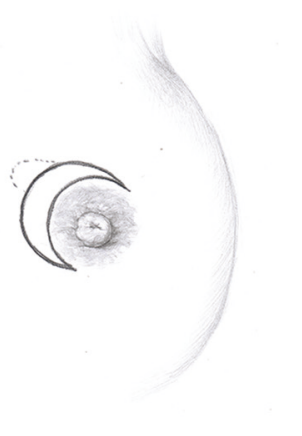

D

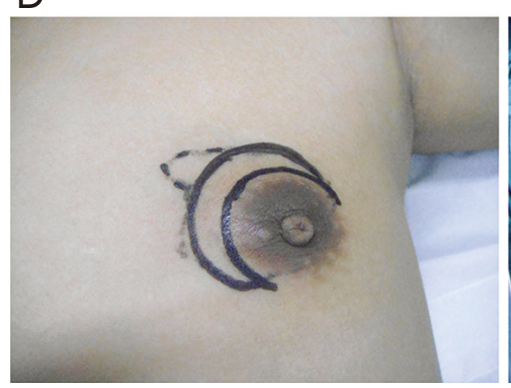

B

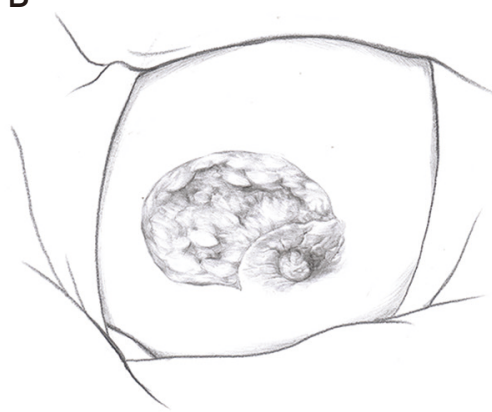

$\mathrm{E}$

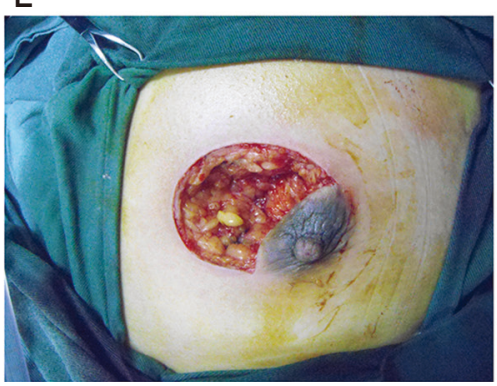

C

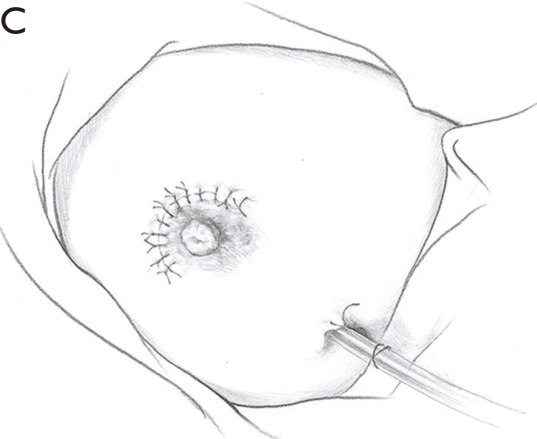

$\mathrm{F}$

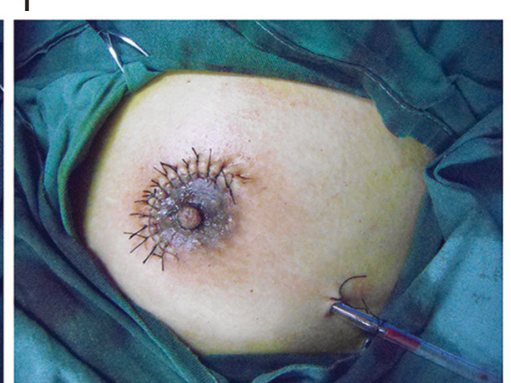

Figure 1 The upper and lower rows represent the schematic and surgical diagrams of the operative procedure for pattern 1 (crescent-shaped incision). (A,D) Outline of the crescent-shaped incision. (B,E) Breast tissue defect after debridement. (C,F) Resurfacing of the breast tissue defect and closure of the wound. Solid line: surgical incision; dotted line: lesion area.

technique was used for each group. Because the breast size varies from one woman to the other, the patterns for large lesions are based on the lesion size relative to the breast size.

\section{Pattern 1: crescent-shaped incision}

This pattern was suitable for small lesions $(\leq 3 \mathrm{~cm})$ beneath or adjacent to the areola. After the complete debridement of the inflamed tissues, the breast defect was usually relatively small. The mammary tissue at the distal end was freed and pulled to repair the defect and wound beneath the areola (Figure 1).

\section{Pattern 2: T-shaped incision}

This pattern was suitable for medium-sized lesions for which the defect could not be closed by the crescent-shaped incision, but the extent of the lesion was no larger than onethird of the whole breast size. In these cases, two assisting periareolar incisions were performed to free mammary tissue around the defect and to pull it inside the defect to repair the wound (Figure 2).

\section{Pattern 3: pear-shaped incision}

This pattern was suitable for lesions larger than one-third of the whole breast size, but not spreading to the other side of the areola. In these cases, the breast defect was larger than those of Pattern 2 and could not be closed by a T-shaped incision. An additional oblique incision was performed on the skin of the mammary tissue-rich area. In this way, new breast tissue flaps could be produced to repair the large defect (Figure 3).

\section{Pattern 4: butterfly-shaped incision}

This pattern was suitable for lesions that extended on both sides of the areola, but for which the total volume of the lesion was less than half of the breast. In these cases, after debridement of the inflamed tissues beneath and around the areola, breast tissue flaps were designed and harvested on both sides and pulled together to repair the defect (Figure 4). 
A

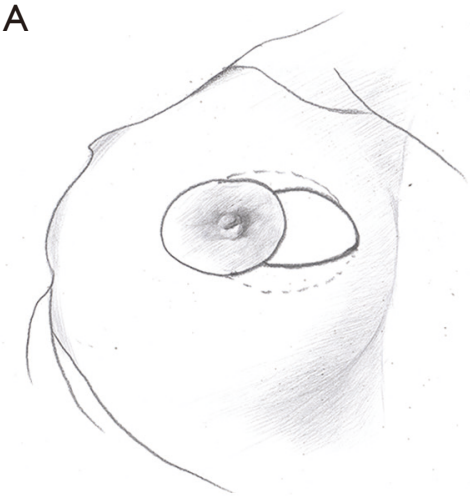

D

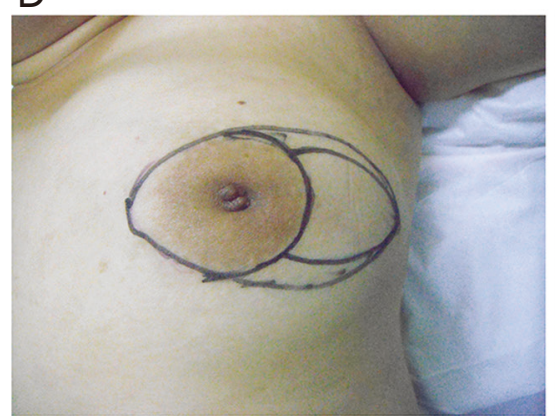

B

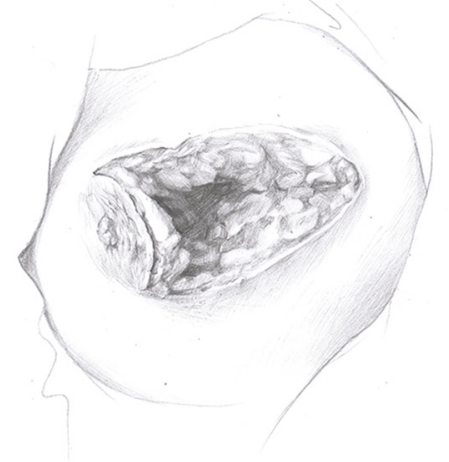

E

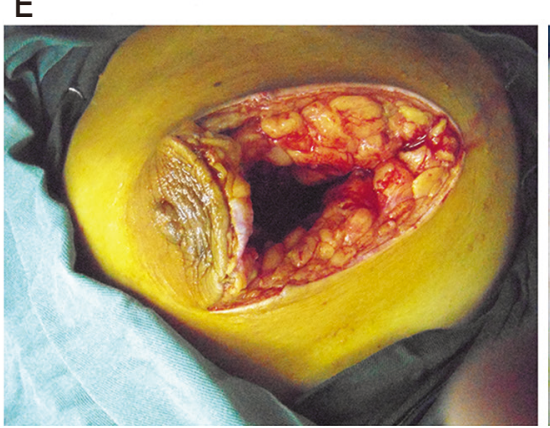

C

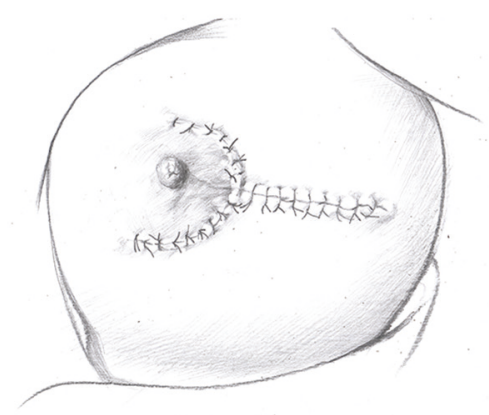

$\mathrm{F}$

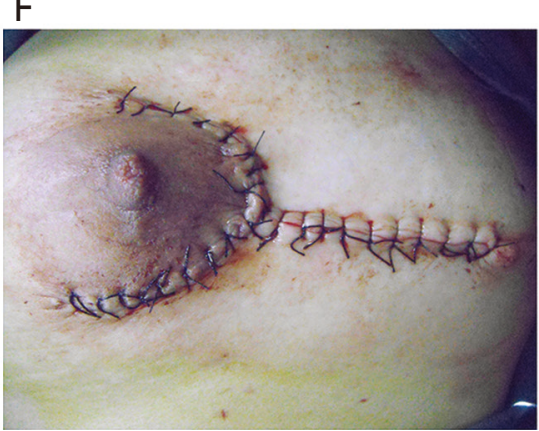

Figure 2 The upper and lower rows represent the schematic and surgical diagrams of the operative procedure for pattern 2 (T-shaped incision). (A,D) Outline of the T-shaped incision. (B,E) Breast tissue defect after debridement. (C,F) Resurfacing of the breast tissue defect and closure of the wound. Solid line: surgical incision; dotted line: lesion area.

\section{$B D G F$}

An ipsilateral random BDGF that contained breast tissue of full-thickness was outlined and freed from the breast region with relatively rich mammary glands. The flap needed to be of an adequate size with a tip angle of no less than $30^{\circ}$ to ensure no torsion or tension. The flap was mobilized from the ipsilateral pectoralis major muscle and transferred to fit the breast defect. The mobile end of the BDGF was pulled directly to the distal margin of the defect without excessive tension. The wound was repeatedly washed before being closed, and then a suction drain was placed. All patients were given empirical prophylactic antibiotics with $1.5 \mathrm{~g}$ cefuroxime on the skin excision. All specimens were subjected to culture. The antibiotic was continued for 3 days if the bacterial culture results were positive.

\section{Postoperative follow-up}

All participants were followed at 6, 12, and 24 months after surgery. Recurrence was evaluated based on physical examination combined with ultrasound and/or mammography, if necessary. At the end of the $2^{\text {nd }}$ year, disease recurrence and complications got evaluated by a research nurse. The change in cosmetic appearance was self-evaluated as "much better", "a little better" or "same or worse" based on the postoperative shape at 2 years after surgery compared with preoperative condition. The improvement in dressing change and bathing was selfevaluated as "improved a lot", "improved a little" or "not improved or getting worse" at 2 years after surgery compared with preoperative condition.

\section{Outcomes}

Operative data (time, blood loss, and intraoperative complications), primary healing time, recurrence, complications during follow-up, and patient-reported outcomes (cosmetic outcome, and improvement in dressing change and bathing) at 2 years after surgery were evaluated. Possible intraoperative and postoperative complications after general anesthesia such as cerebrovascular events, shock, allergy, blood loss $>500 \mathrm{~mL}$, or any event that could 
A

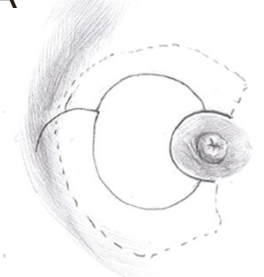

$\mathrm{F}$

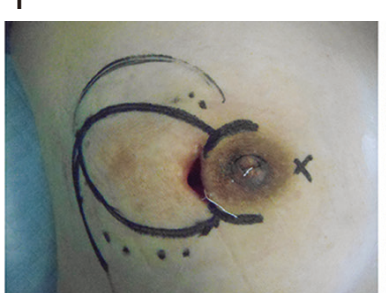

B

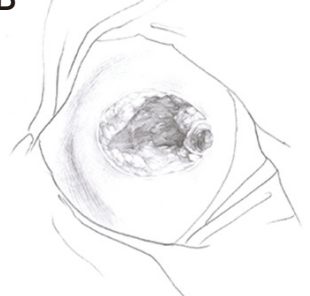

G

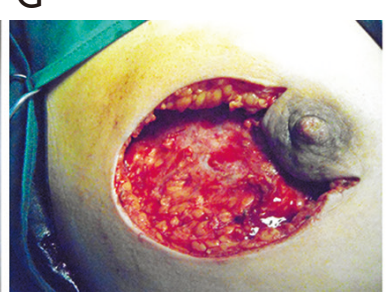

C
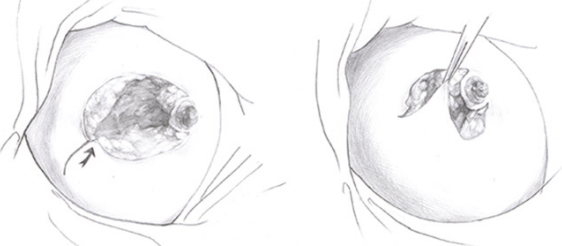

$\mathrm{H}$

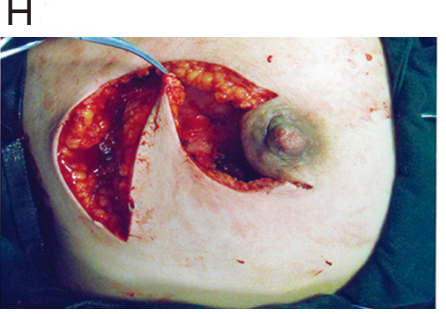

$\mathrm{E}$

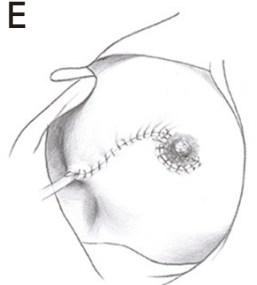

I

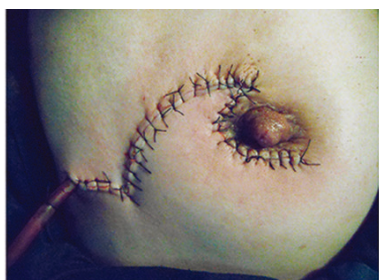

Figure 3 The upper and lower rows represent the schematic and surgical diagrams of the operative procedure for pattern 3 (pear-shaped incision). (A,F) Outline of the pear-shaped incision. (B,G) Breast tissue defect after debridement. (C,D,H) Retraction of the flap toward the contralateral margin. (E,I) Resurfacing of the breast tissue defect and closure of the wound. Solid line: surgical incision; dotted line: lesion area.

A
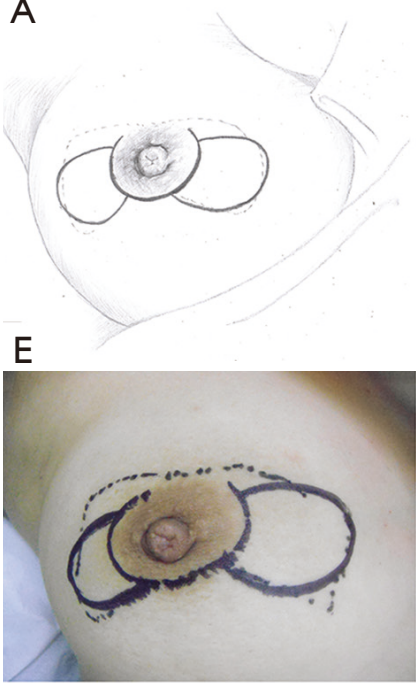

B

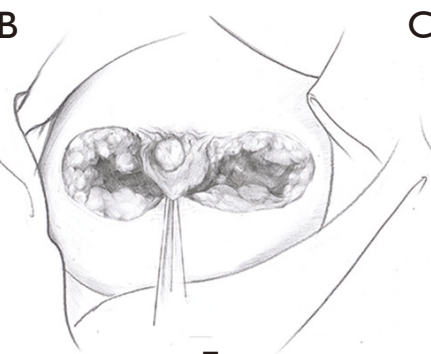

$\mathrm{F}$

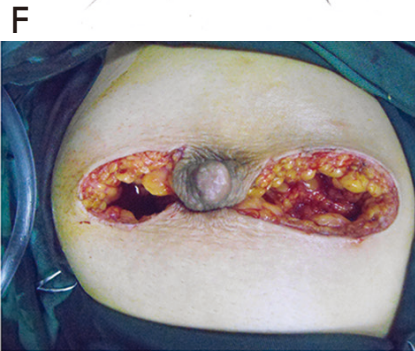

C

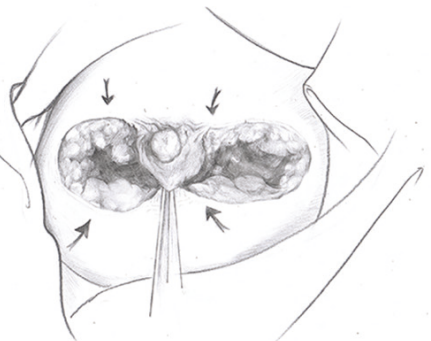

G
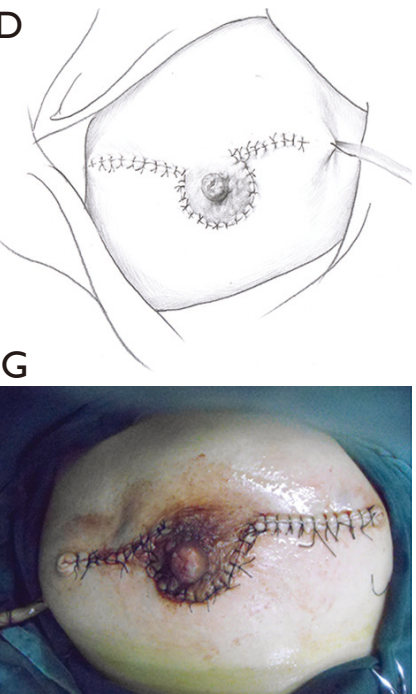

Figure 4 The upper and lower rows represent the schematic and surgical diagrams of the operative procedure for pattern 4 (butterfly-shaped incision). (A,E) Outline of the butterfly-shaped incision. (B,C,F) Harvest and mobilization of the BDGF. (D,G) Closure of the wound. Solid line: surgical incision; dotted line: lesion area. BDGF, breast dermo-glandular flap.

lead to death, prolonged hospitalization, or permanent impairment got evaluated.

\section{Statistical analysis}

Categorical data are presented as numbers and percentages.
Continuous variables are presented as medians (range) or as means \pm standard deviation, as appropriate based on the Kolmogorov-Smirnov test. Differences among groups were evaluated using ANOVA and the least significant difference (LSD) post hoc test. Statistical analysis was carried out using SPSS 16.0 (SPSS Inc., Chicago, IL, USA). P values 
Table 1 Characteristics of the patients with recurrent/refractory IGM

\begin{tabular}{|c|c|}
\hline Variables & Patients $(n=68)$ \\
\hline \multicolumn{2}{|l|}{ Age (years), n (\%) } \\
\hline $18-35$ & $37(54.4)$ \\
\hline $35-50$ & $29(42.7)$ \\
\hline$>50$ & $2(2.9)$ \\
\hline \multicolumn{2}{|l|}{ Body mass index $\left(\mathrm{kg} / \mathrm{m}^{2}\right), \mathrm{n}(\%)$} \\
\hline$<18.5$ & 0 \\
\hline $18.5-23.9$ & $17(25.0)$ \\
\hline $24-26.9$ & $27(39.7)$ \\
\hline$>27$ & $24(35.3)$ \\
\hline \multicolumn{2}{|l|}{ Duration of IGM (months), n (\%) } \\
\hline $3-6$ & $12(17.6)$ \\
\hline $7-12$ & $22(32.4)$ \\
\hline$>12$ & $34(50.0)$ \\
\hline \multicolumn{2}{|l|}{ Clinical manifestation, $\mathrm{n}(\%)$} \\
\hline Nipple discharge & $8(11.8)$ \\
\hline Duct fistula & $21(30.9)$ \\
\hline Periareolar abscess & $29(42.6)$ \\
\hline Breast mass $(>3 \mathrm{~cm})$ & $51(75.0)$ \\
\hline \multicolumn{2}{|l|}{ History of treatment, n (\%) } \\
\hline Corticosteroid & $46(67.6)$ \\
\hline Antimicrobial therapy & $42(61.8)$ \\
\hline Surgical drainage & $40(58.8)$ \\
\hline Surgical excision & $37(54.4)$ \\
\hline Smoking, $\mathrm{n}(\%)$ & $7(10.3)$ \\
\hline Drinking, n (\%) & $5(7.4)$ \\
\hline Trauma within 1 month, $\mathrm{n}(\%)$ & $8(11.8)$ \\
\hline Diabetes mellitus, n (\%) & $7(10.3)$ \\
\hline Hypertension, n (\%) & $12(17.6)$ \\
\hline
\end{tabular}

IGM, idiopathic granulomatous mastitis.

$<0.05$ were considered statistically significant.

\section{Results}

\section{Characteristics of the patients}

During the study period, 491 patients were diagnosed with IGM. Of the 491 patients, 293 (59.7\%) responded to conservative treatments such as steroids, and abscess incision and drainage, 130 (26.5\%) were cured after simple excision, and 68 (13.8\%) of them ultimately required further surgery and were included in the present study. The clinical characteristics of the patients with recurrent/ refractory IGM are presented in Table 1. A total of 68 consecutive patients with a median age of 35 (range, 22 to $55)$ years were enrolled. The duration of the IGM was 3 to 22 months. The median size of the lesions was 3.5 (range, 0.9 to 9.1$) \mathrm{cm}$.

\section{Operative data}

According to the location and extent of the lesions, the patients were divided into four patterns, with $21,19,16$, and 12 patients in patterns $1,2,3$, and 4, respectively (Table 2). As the lesion size increased, the operation time was significantly prolonged, and blood loss was more important (both $\mathrm{P}<0.05$ ). No complications occurred during surgery. Positive bacterial culture results were found in two patients with pattern 1 (one Streptococcus and one Corynebacterium), two patients in pattern 2 (one Staphylococcus and one Corynebacterium), one patient in pattern 3 (Staphylococcus), and two patients in pattern 4 (two Corynebacterium).

\section{Follow-up}

All patients were followed for 2 years. The flaps survived without any complications. The wounds healed by primary intention within 2 weeks after surgery. During the 2-year follow-up, the recurrence of IGM was observed in three patients. They were successfully treated with a second operation. No patients complained of severe pain, skin numbness, or other complications. The repaired breast had a satisfactory shape and was approximately symmetric to the other breast. The postoperative self-reported cosmetic result compared with preoperative condition was "much better" in 45 patients (66.2\%), "a little better" in 18 (26.5\%), and "same or worse" in five $(7.3 \%)$. The unfavorable cosmetic outcome was mainly reported in patterns 3 and 4. The self-reported improvement in dressing change and bathing compared with preoperative condition was "improved a lot" in 51 patients (75.0\%), "improved a little" in $11(16.2 \%)$, and "not improved or getting worse" in six (8.8\%). Among the six patients who reported "not improved or getting worse" on dressing change and bathing, there were two, two, and two patients in patterns 2,3 , and 4 , 
Table 2 Clinical characteristics by different patterns

\begin{tabular}{|c|c|c|c|c|}
\hline Variable & Pattern $1(n=21)$ & Pattern $2(n=19)$ & Pattern $3(n=16)$ & Pattern $4(n=12)$ \\
\hline Operative time $(\min )^{*}$ & $47 \pm 12$ & $88 \pm 23$ & $126 \pm 31$ & $166 \pm 39$ \\
\hline Blood loss $(\mathrm{mL})^{*}$ & $35 \pm 6$ & $66 \pm 28$ & $144 \pm 60$ & $256 \pm 93$ \\
\hline $\begin{array}{l}\text { Positive bacterial } \\
\text { culture, } \mathrm{n}(\%)\end{array}$ & $2(9.5)$ & $2(10.5)$ & $1(6.3)$ & $2(16.7)$ \\
\hline
\end{tabular}

${ }^{*} \mathrm{P}<0.05$ among groups.

Table 3 Patient-reported outcomes at 2 years after surgery compared with preoperative condition

\begin{tabular}{|c|c|c|c|c|c|}
\hline Variable & Pattern $1(n=21)$ & Pattern $2(n=19)$ & Pattern $3(n=16)$ & Pattern $4(n=12)$ & All $(n=68)$ \\
\hline Much better & $18(85.7)$ & $12(63.2)$ & $9(56.3)$ & $6(50.0)$ & $45(66.2)$ \\
\hline A little better & $3(14.3)$ & $6(31.6)$ & $5(31.2)$ & 4 (33.3) & $18(26.5)$ \\
\hline Same or worse & 0 & $1(5.3)$ & $2(12.5)$ & $2(16.7)$ & $5(7.3)$ \\
\hline \multicolumn{6}{|l|}{ Dressing change and bathing, $\mathrm{n}(\%)$} \\
\hline Improved a lot & $20(95.2)$ & $13(68.4)$ & $10(62.5)$ & $8(66.7)$ & $51(75.0)$ \\
\hline Improved a little & $1(4.8)$ & $4(21.1)$ & $4(25.0)$ & $2(16.7)$ & 11(16.2) \\
\hline Not improved or getting worse & 0 & $2(10.5)$ & $2(12.5)$ & $2(16.7)$ & $6(8.8)$ \\
\hline
\end{tabular}

respectively (Table 3).

\section{Discussion}

Resection can be performed for IGM, but recurrence and tissue defects remain issues. Here we reported our 6-year experience with systematic four-pattern surgical methods that include the use of a random BDGF. The results strongly suggest that these systematic patterns based on BDGF are appropriate to treat patients with IGM. The recurrence rate was low, there were no complications during surgery, and the cosmetic results and improvement in dressing change and bathing were generally favourable.

In the present study, only seven (10.3\%) patients were smokers, mainly because women represent a small proportion of the smokers in China (29), and smoking was probably not associated with the onset or recurrence of IGM, as supported by a previous report (30). On the other hand, some other studies reported that smoking could significantly increase the risk of IGM recurrence $(16,31,32)$. The present study was not designed to study the epidemiology of IGM since only the patients who underwent surgery were included. Additional studies are necessary to examine this issue.

Although IGM is benign, it is not cured easily, and recurrence is common, imposing an important physical and psychological burden on the patients (27). Only a small number of patients with early-stage IGM achieve good curative effect. Many patients are treated by an improper surgical approach, possibly leading to disease recurrence in some cases, or even breast deformity or mastectomy in the worst cases $(5,6)$. Since incomplete excision will lead to recurrence, an extended excision is a favorable option $(5,6)$, but has a higher likelihood to lead to healing problem and breast deformity. In the present study, an extended excision was first performed to remove the entire lesion, 
including the involved skin, abscess, fistula, pathologic ducts, inflammatory debris, and necrotic tissues. Among the 68 patients, only three (4\%) had recurrence, which is in the lower range of the reported recurrence rate of $0-38 \%$ after surgery (16-18,23-26). The three patients with recurrence were in patterns 3 and 4, which represent large lesions encompassing a significant proportion of the breast and, therefore, the likelihood of leaving occult and subclinical disease foci is probably higher than in patterns 1 and 2 . The low recurrence rate observed in the present study could be due to a systematic assessment of the affected breast and careful operation to remove all visible lesions.

Since the breasts are important to the women's image, the surgeons are faced with two opposite goals: (I) the entire removal of the lesions; and (II) a favorable cosmetic outcome (33). To prevent recurrence, an area as large as possible around the lesions should be removed. On the other hand, from a cosmetic point of view, smaller resections will lead to better cosmetic outcomes. The surgeon has to carefully balance these two conflicting aspects to satisfy their patients (34). Extended excision may lead to a large tissue defect, which is difficult to close, and an unfavorable cosmetic outcome may result, eventually leading to a mastectomy in some cases $(22,23)$. To solve this surgical challenge, we previously reported a technique to repair surgical breast defects, in which a random BDGF is used to fill the defect (28). In patients with IGM, the extent of the defect will vary widely among patients because of the variable extent of the disease. Therefore, we divided the patients into four different patterns according to the location and extent of the lesion, and each pattern was suitable for a specific surgical technique, using either crescent-shaped, T-shaped, pear-shaped, and butterflyshaped incisions. As for cosmetic outcome, $66.2 \%$ of the patients were satisfied with the outcome and evaluated the outcome as "much better". The proportion of "much better" and "a little better" combined was as high as $92.7 \%$, which confirms the feasibility and advantage of the use of a BDGF for the surgical management of IGM. The patients who evaluated their cosmetic results as "same or worse" were mainly in patterns 3 and 4, which could be attributed to large surgical extension and severe breast deformity.

For patients with severe inflammatory lesions, cosmetic appearance is not the only evaluating criterion for surgical outcomes. Because of the sufferings from severe inflammatory lesions, these patients are more concerned with restoring a normal life rather than the cosmetic appearance. This indicator is not widely used in studies on IGM, but we believe that it might be a comprehensive assessment of a patient's general well-being (35). The dressing change and bathing was generally improved in most patients after surgery, which was a comprehensive evaluation of their wounds healing, recurrence, and return to the normal activities of daily living. Indeed, the dressing change and bathing was improved a lot by $75.0 \%$ of the patients. Since all patients included in the present study sought a definitive surgery because of recurring IGM after conservative and surgical treatment, we believe that an individualized surgical approach might be applied for effective patient management. The previous studies of wide excision of IGM lesions are small series that did not examine satisfaction. Only Ahmed et al. (36) showed that the application of therapeutic mammoplasty techniques during the surgical management of IGM with mild-to-moderate symptoms led to $77 \%$ satisfaction. In the present study, a similar level of satisfaction was achieved in IGM patients with varying severity of symptoms who underwent the systematic four-pattern surgery after failure of conservative or primary surgical treatment.

The four surgical patterns introduced in this study represented all the patients we encountered at our clinic during the study period. We cannot exclude the possibility of some patients with a special condition and not fitting those four patterns. For patients with particularly serious and extended lesions, conservative treatment and drainage may make some lesions smaller and allow surgery, but if the lesions still affect the whole breast, the four patterns are not applicable, and the only remaining option is mastectomy. The four patterns determined here are purely for surgical management. Freeman et al. (5) proposed an algorithm that includes the conservative management of IGM, and it could be interesting to investigate whether patterns of conservative and surgical management could be merged. Additional multicenter studies are necessary to validate this classification and determine the course in special cases.

This study has limitations. First, the sample size was small and from a single center. Second, the follow-up was relatively short. The median time to recurrence was 280 days (range, 164-358 days) in the study by Chirappapha et al. (7). Although the patients in the present study were followed for 2 years, this might still not be long enough because recurrence might occur after 2 years. Finally, no control group of patients treated conservatively or using traditional surgery without the application of the four patterns was included.

In conclusion, for patients with IGM and a refractory 
response to conservative therapy, or for those having unsatisfactory outcome after primary surgery, we established a four-pattern surgical management strategy based on the lesion extent and location and used a BDGF to fill the surgical defect. In general, the surgical and patient-reported outcomes were satisfactory. Recurrences and unsatisfactory results were mainly attributable to large lesions. Using extended excision patterns and BDGF, we can close the wound and repair large breast defects, thus achieving a satisfactory cosmetic outcome and improvement in dressing change and bathing for patients with IGM.

\section{Acknowledgments}

Funding: None.

\section{Footnote}

Conflicts of Interest: All authors have completed the ICMJE uniform disclosure form (available at http://dx.doi. org/10.21037/gs.2020.02.06). The authors have no conflicts of interest to declare.

Ethical Statement: The authors are accountable for all aspects of the work in ensuring that questions related to the accuracy or integrity of any part of the work are appropriately investigated and resolved. The study was approved by the ethics committee of the PUMCH. All patients signed an informed consent form. The study was approved by the ethics committee of the PUMCH. All patients signed an informed consent form.

Open Access Statement: This is an Open Access article distributed in accordance with the Creative Commons Attribution-NonCommercial-NoDerivs 4.0 International License (CC BY-NC-ND 4.0), which permits the noncommercial replication and distribution of the article with the strict proviso that no changes or edits are made and the original work is properly cited (including links to both the formal publication through the relevant DOI and the license). See: https://creativecommons.org/licenses/by-nc-nd/4.0/.

\section{References}

1. Kessler E, Wolloch Y. Granulomatous mastitis: a lesion clinically simulating carcinoma. Am J Clin Pathol 1972;58:642-6.

2. Zhou F, Yu LX, Ma ZB, et al. Granulomatous lobular mastitis. Chronic Dis Transl Med 2016;2:17-21.

3. Kasales CJ, Han B, Smith JS Jr, et al. Nonpuerperal mastitis and subareolar abscess of the breast. AJR Am J Roentgenol 2014;202:W133-9.

4. Barreto DS, Sedgwick EL, Nagi CS, et al. Granulomatous mastitis: etiology, imaging, pathology, treatment, and clinical findings. Breast Cancer Res Treat 2018;171:527-34.

5. Freeman CM, Xia BT, Wilson GC, et al. Idiopathic granulomatous mastitis: A diagnostic and therapeutic challenge. Am J Surg 2017;214:701-6.

6. Wilson JP, Massoll N, Marshall J, et al. Idiopathic granulomatous mastitis: in search of a therapeutic paradigm. Am Surg 2007;73:798-802.

7. Chirappapha P, Thaweepworadej P, Supsamutchai C, et al. Idiopathic granulomatous mastitis: A retrospective cohort study between 44 patients with different treatment modalities. Ann Med Surg (Lond) 2018;36:162-7.

8. Baslaim MM, Khayat HA, Al-Amoudi SA. Idiopathic granulomatous mastitis: a heterogeneous disease with variable clinical presentation. World J Surg 2007;31:1677-81.

9. Leong PW, Chotai NC, Kulkarni S. Imaging Features of Inflammatory Breast Disorders: A Pictorial Essay. Korean J Radiol 2018;19:5-14.

10. Lacambra M, Thai TA, Lam CC, et al. Granulomatous mastitis: the histological differentials. J Clin Pathol 2011;64:405-11.

11. Bakaris S, Yuksel M, Ciragil P, et al. Granulomatous mastitis including breast tuberculosis and idiopathic lobular granulomatous mastitis. Can J Surg 2006;49:427-30.

12. Li J. Diagnosis and Treatment of 75 Patients with Idiopathic Lobular Granulomatous Mastitis. J Invest Surg 2019;32:414-20.

13. Sheybani F, Naderi HR, Gharib M, et al. Idiopathic granulomatous mastitis: Long-discussed but yet-to-beknown. Autoimmunity 2016;49:236-9.

14. Benson JR, Dumitru D. Idiopathic granulomatous mastitis: presentation, investigation and management. Future Oncol 2016;12:1381-94.

15. Pandey TS, Mackinnon JC, Bressler L, et al. Idiopathic granulomatous mastitis--a prospective study of 49 women and treatment outcomes with steroid therapy. Breast J 2014;20:258-66.

16. Yabanoglu H, Colakoglu T, Belli S, et al. A Comparative Study of Conservative versus Surgical Treatment Protocols for 77 Patients with Idiopathic Granulomatous Mastitis. 
Breast J 2015;21:363-9.

17. Shin YD, Park SS, Song YJ, et al. Is surgical excision necessary for the treatment of Granulomatous lobular mastitis? BMC Womens Health 2017;17:49.

18. Pereira FA, Mudgil AV, Macias ES, et al. Idiopathic granulomatous lobular mastitis. Int J Dermatol 2012;51:142-51.

19. Lei X, Chen K, Zhu L, et al. Treatments for Idiopathic Granulomatous Mastitis: Systematic Review and MetaAnalysis. Breastfeed Med 2017;12:415-21.

20. Sheybani F, Sarvghad M, Naderi HR, et al. Treatment for and clinical characteristics of granulomatous mastitis. Obstet Gynecol 2015;125:801-7.

21. Hovanessian Larsen LJ, Peyvandi B, Klipfel N, et al. Granulomatous lobular mastitis: imaging, diagnosis, and treatment. AJR Am J Roentgenol 2009;193:574-81.

22. Kiyak G, Dumlu EG, Kilinc I, et al. Management of idiopathic granulomatous mastitis: dilemmas in diagnosis and treatment. BMC Surg 2014;14:66.

23. Akcan A, Akyildiz H, Deneme MA, et al. Granulomatous lobular mastitis: a complex diagnostic and therapeutic problem. World J Surg 2006;30:1403-9.

24. Kok KY, Telisinghe PU. Granulomatous mastitis: presentation, treatment and outcome in 43 patients. Surgeon 2010;8:197-201.

25. Mathew M, Siwawa P, Misra S. Idiopathic granulomatous mastitis: an inflammatory breast condition with review of the literature. BMJ Case Rep 2015;2015.

26. Azlina AF, Ariza Z, Arni T, et al. Chronic granulomatous mastitis: diagnostic and therapeutic considerations. World J Surg 2003;27:515-8.

27. Uysal E, Soran A, Sezgin E, et al. Factors related to recurrence of idiopathic granulomatous mastitis: what do we learn from a multicentre study? ANZ J Surg 2018;88:635-9.

Cite this article as: Zhang X, Li Y, Zhou Y, Liu D, Chen L, Niu K, Sun Q, Huang H. A systematic surgical approach for the treatment of idiopathic granulomatous mastitis: a case series. Gland Surg 2020;9(2):261-270. doi: 10.21037/gs.2020.02.06
28. Zhang X, Lin Y, Sun Q, et al. Dermo-glandular flap for treatment of recurrent periductal mastitis. J Surg Res 2015;193:738-44.

29. Wang M, Luo X, Xu S, et al. Trends in smoking prevalence and implication for chronic diseases in China: serial national cross-sectional surveys from 2003 to 2013. Lancet Respir Med 2019;7:35-45.

30. Gautham I, Radford DM, Kovacs CS, et al. Cystic neutrophilic granulomatous mastitis: The Cleveland Clinic experience with diagnosis and management. Breast J 2019;25:80-5.

31. Dixon JM, Ravisekar O, Chetty U, et al. Periductal mastitis and duct ectasia: different conditions with different aetiologies. Br J Surg 1996;83:820-2.

32. Co M, Cheng VCC, Wei J, et al. Idiopathic granulomatous mastitis: a 10-year study from a multicentre clinical database. Pathology 2018;50:742-7.

33. Bertozzi N, Pesce M, Santi PL, et al. Oncoplastic breast surgery: comprehensive review. Eur Rev Med Pharmacol Sci 2017;21:2572-85.

34. Hladik M, Schoeller T, Ensat F, et al. Idiopathic granulomatous mastitis: successful treatment by mastectomy and immediate breast reconstruction. J Plast Reconstr Aesthet Surg 2011;64:1604-7.

35. McCarthy CM, Cano SJ, Klassen AF, et al. The magnitude of effect of cosmetic breast augmentation on patient satisfaction and health-related quality of life. Plast Reconstr Surg 2012;130:218-23.

36. Ahmed YS, Abd El Maksoud W. Evaluation of therapeutic mammoplasty techniques in the surgical management of female patients with idiopathic granulomatous mastitis with mild to moderate inflammatory symptoms in terms of recurrence and patients' satisfaction. Breast Dis 2016;36:37-45. 